\title{
OBÉPINE : UNE EXPÉRIENCE FRANÇAISE DE SUIVI DE L'ÉPIDÉMIE DE COVID-19 À TRAVERS LES EAUX USÉES
}

\author{
OBEPINE: A FRENCH EXPERIMENT TO MONITOR THE COVID-19 \\ EPIDEMIC THROUGH WASTEWATER
}

\begin{abstract}
Vincent MARECHAL ${ }^{1^{*}}$, Laurent MOULIN ${ }^{2 *}$, Yvon MADAY ${ }^{3 *}$, Mickaël BONI 4*, Rémy TEYSSOU ${ }^{4}$, Sébastien WURTZER ${ }^{2}$, Jean-Marie MOUCHEL ${ }^{5}$, Isabelle BERTRAND ${ }^{6}$, Christophe GANTZER ${ }^{6}$, Soizick LE GUYADER ${ }^{7}$

*Contribution égale
\end{abstract}

(Manuscrit reçu le 7 octobre 2021 et accepté le 19 octobre 2021)

\begin{abstract}
RÉSUMÉ
Le consortium de recherche Obépine (Observatoire Épidémiologique dans les Eaux Usées) s'est attaché, depuis mars 2020, à exploiter la présence du SARS-CoV-2 dans les eaux usées pour proposer un suivi épidémiologique de la COVID-19 en France. Bien que l'épidémiologie des eaux usées soit une discipline déjà ancienne, elle n'a jamais connu de déploiement aussi important à l'échelle nationale et internationale. Réunissant des équipes de recherche aux compétences variées, Obépine a établi les premiers protocoles de quantification du génome viral dans eaux usées des stations de traitement des eaux usées (STEU). Le réseau sentinelle qui a été construit avec l'appui du Ministère français de l'Enseignement supérieur, de la Recherche et de l'Innovation comprend à ce jour 200 STEU en France métropolitaine et outre-mer. II permet d'évaluer de façon bihebdomadaire la dynamique de l'épidémie dans plus de $33 \%$ de la population française. Cet article présente la genèse d'un projet de surveillance et les axes de recherche d'un réseau qui souhaite s'attacher - dans un futur proche - à compléter les dispositifs d'alerte précoce et de suivi des infections endémiques ou émergentes existants.

Mots-clés : SARS-CoV-2, COVID-19, épidémiologie dans les eaux usées, réseau sentinelle
\end{abstract}

\begin{abstract}
Since March 2020, the Obépine (Observatoire Epidémiologique dans les Eaux Usées) research consortium has been working on exploiting the presence of SARS-COV-2 in wastewater to propose epidemiological monitoring of COVID-19 in France. Although wastewater epidemiology is a long-standing discipline, it has never been so widely deployed on a national and international scale. Bringing together research teams with varied skills, Obépine has established the first protocols for quantifying the viral genome in samples from wastewater treatment plants (WWTPs). The sentinel network, which was built with the support of the French Ministry of Higher Education, Research and Innovation, currently includes 200 WWTPs in mainland France and overseas. It allows the bi-weekly assessment of the dynamics of the epidemic in more than $33 \%$ of the French population. This paper presents the genesis of a surveillance project and the lines of research of a network that wishes to work - in the near future - to complete the existing early warning and monitoring systems for endemic or emerging infections.

Key-Words: SARS-CoV-2, COVID-19, wastewater epidemiology, sentinel network
\end{abstract}

\footnotetext{
1- Sorbonne Université, INSERM, Centre de Recherche Saint-Antoine, Paris, France

2- Eau de Paris, R\&D Laboratory, DRDQE Ivry/Seine, France

3- Sorbonne Université, CNRS, Université de Paris, Laboratoire Jacques-Louis Lions (LLLL), et Institut Universitaire de France, Paris, France

4- Institut de recherche biomédicale des armées, Brétigny-sur-Orge, France

5- Sorbonne Université, CNRS, EPHE, UMR 7619 Metis, e-LTER Zone Atelier Seine, Paris, France

6- Université de Lorraine, CNRS, LCPME, F-54000, Nancy, France

7- Ifremer, laboratoire de Microbiologie, SG2M/LSEM, BP 21105, 44311 Nantes, France
} 


\section{INTRODUCTION}

La surveillance des maladies infectieuses - dans leur composante humaine et/ou zoonotique - est un élément central de la prévention et du contrôle des épidémies. Le concept de santé globale/une seule santé (angl. One Health) rappelle que la circulation des pathogènes est étroitement liée aux relations complexes qu'entretiennent l'Homme, le monde animal et l'environnement (Zinsstag et al. 2018 ; Di Marco et al. 2020). Cette vision holistique de l'infectiologie a permis de souligner que les émergences infectieuses sont largement déterminées par des facteurs socio-économiques, agronomiques, climatiques et/ou écologiques. Dès 2008, Jones et collaborateurs avaient déjà rappelé que l'essentiel des maladies émergentes chez l'Homme était d'origine animale et qu'elles trouvaient dans près de $75 \%$ des cas leur origine dans la faune sauvage (Jones et al. 2008).

À l'interface entre l'Homme, les animaux et l'environnement, les eaux usées constituent par essence une ressource d'intérêt majeur pour la surveillance des agents pathogènes et de leur évolution, mais également pour la surveillance des résistances aux molécules antimicrobiennes (Mao et al. 2021). Beaucoup d'autres molécules, témoins de l'exposition de l'Homme aux pesticides, aux drogues, aux traitements médicaux y sont régulièrement recherchées, contribuant ainsi à mieux caractériser l'exposome et à identifier facilement les risques sanitaires à l'échelle locale (Lorenzo et Picó, 2019 ; Wild, 2005 ; Devault et Karolak, 2020 ; Devault et al. 2018). Réceptacle des excréments humains, parfois animaux, les eaux usées constituent tout à la fois un vecteur privilégié dans la transmission des maladies féco-orales et une matrice de choix pour établir une cartographie dynamique des agents pathogènes en circulation sur un territoire donné et suivre leur incidence saisonnière (Prevost et al. 2015 ; Prado et al. 2019 ; Le Guyader et al. 2014 ; Mao et al. 2020 ; O'Brien et Xagoraraki, 2019 ; Corpuz et al. 2020 ; Wurtzer v. 2021a). L'analyse microbiologique des eaux usées a progressivement démontré son potentiel, encore largement sous-exploité, comme dispositif de surveillance et d'alerte précoce des émergences infectieuses (Mao et al. 2020). L'initiative portée par les équipes de recherche réunies autour du projet Obépine (OBservatoire ÉPIdémiologique daNs les Eaux usées) est l'illustration du succès d'une approche multidisciplinaire intégrée qui a permis à la France de mettre en place en quelques mois seulement un dispositif de surveillance de l'épidémie de COVID-19 via l'analyse des eaux usées. Cette stratégie pourrait se généraliser dans un avenir proche, notamment dans le but d'évaluer l'état de santé des populations dans le bassin versant d'une station de traitement des eaux usées (STEU), voire à s'étendre à d'autres questions en lien avec l'infectiologie ou la toxicologie environnementale.

\section{POURQUOI L'ÉPIDÉMIE DE COVID-19 A-T-ELLE PU ÊTRE SUIVIE DANS LES EAUX USÉES ?}

Le SARS-CoV-2 est l'agent étiologique de la COVID-19 («Coronavirus disease 2019»), une maladie à point d'entrée respiratoire et dont l'évolution peut être fatale notamment chez les sujets âgés ou atteints de comorbidités (Hu et al. 2021). Initialement identifiée à Wuhan - capitale de la province du Hubei (Chine) en décembre 2019, l'épidémie s'est rapidement étendue au point d'être qualifiée de pandémie par l'OMS dès mars 2020 (Zhu et al. 2020). À ce jour, l'infection a été officiellement détectée chez plus de 200 millions de patients et aurait entraîné près de 4,5 millions de morts (site OMS, consulté le 20 août 2021).

Le SARS-CoV-2 appartient aux Coronaviridae, une famille de virus à ARN positif, enveloppés, dont 6 membres avaient déjà été identifiés chez l'Homme (V'kovski et al. 2021). Produits d'une émergence zoonotique déjà ancienne, quatre d'entre eux (HCoV-229E, HCoV-NL63, HCoV-OC43 et HCoV-HKU1) sont parfaitement adaptés à l'Homme, chez lequel ils circulent selon un mode saisonnier hivernal en causant le plus souvent des infections respiratoires hautes bénignes ou modérées (Paules et al. 2020). Des ancêtres de ces virus ont été identifiés chez des rongeurs et des chauves-souris (Corman et al. 2018), témoignant d'une origine animale probable. Trois autres coronavirus humains - SARS-CoV, MERS-CoV et SARS-CoV-2 - sont d'émergence zoonotique récente et associés à des atteintes parfois létales (15\%, $35 \%$ et 1,5\% respectivement) (Ioannidis, 2021).

Le SARS-CoV-2 est transmis principalement par contact direct ou indirect, par l'intermédiaire de gouttelettes de salive, de sécrétions ou d'aérosols respiratoires (Meyerowitz et al. 2021). Le manuportage, le transport de virus infectieux via des surfaces inertes ou sur des aliments crus pourraient également contribuer à la circulation du virus dans des proportions qui restent à préciser. Enfin, la présence du virus dans les selles (voir infra) a incité certains auteurs à supposer la possibilité d'une transmission par aérosols fécaux, ce qui reste à établir de façon formelle (McDermott et al. 2020 ; Kang et al. 2020 ; Elsamadony et al. 2021 ; Danchin et al. 2020).

\section{POURQUOI A-T-ON JUGULÉ L'ÉPIDÉMIE DE SARS-COV (2002-2003) EN QUELQUES MOIS SEULEMENT ALORS QUE L'EPIDÉMIE DE SARS-COV-2 N'EST TOUJOURS PAS SOUS CONTRÔLE ?}

La comparaison des épidémies de SRAS (syndrome respiratoire aigu sévère lié au SARS-CoV) (2002-2003) et de COVID-19 montre que les mesures qui avaient permis d'endiguer l'épidémie de SRAS - détection précoce et isolement des cas, mise en quarantaine des contacts, distanciation sociale et, dans certains cas, quarantaine collective - se sont avérées inefficaces pour juguler la pandémie actuelle (Wilder-Smith et al. 2020). Dans le cas du SRAS, la transmission du virus s'opère principalement par l'intermédiaire des patients les plus gravement atteints et seulement après l'apparition des signes cliniques. Cette caractéristique a permis d'identifier et d'isoler rapidement les porteurs sur une base syndromique (fièvre, syndromes respiratoires) et de mettre en quarantaine les personnes contacts avant qu'elles ne transmettent le virus. À l'opposé, pour la COVID-19, l'excrétion virale précède l'apparition des symptômes de quelques jours, rendant la transmission possible avant même que les 
malades ne soient identifiés (Rothe et al. 2020). On estime par ailleurs que les porteurs non symptomatiques étaient assez exceptionnels dans le cas du SRAS et qu'ils ont très peu contribué à faire circuler le virus. À l'opposé, un modèle récent suggère que $59 \%$ de l'ensemble des transmissions proviendraient de porteurs asymptomatiques (35\% provenant d'individus pré-symptomatiques et $24 \%$ d'individus qui ne développent jamais de symptômes) (Johansson et al. 2021). Dans cette hypothèse, la majorité des transmissions se feraient donc à partir de porteurs non symptomatiques ce qui explique l'échec des stratégies d'isolement qui se limitent aux malades (Gao et al. 2021). Le déploiement des tests individuels de masse - d'abord par RT-qPCR puis par recherche des antigènes viraux dans les sécrétions nasales - ont été rendus possibles par l'isolement et le séquençage rapide du SARS-CoV-2. Ils ont sans aucun doute contribué à faciliter l'identification des porteurs et à visualiser depuis juin 2020 en France - la trajectoire de l'épidémie. Toutefois, cette campagne de tests massive est associée à un coût exorbitant. D'après le rapport de la Cour des comptes de juillet 2021 intitulé "Les dépenses publiques pendant la crise et le bilan opérationnel de leur utilisation ", le coût total des tests individuels s'élèverait à 2,8 Md d'euros pour 2020 et les dépenses de ville relatives aux tests pourraient atteindre 4,9 Md d'euros en 2021, sans qu'une réelle surveillance du respect des mesures d'isolement n'ait été mise en place. On peut aussi noter le manque d'homogénéité dans la population testée au cours de l'épidémie, entre l'été 2020 où les tests étaient exceptionnels, la période de Noël où les personnes se sont testées massivement en prévision des rencontres familiales, puis peu pendant les jours fériés, et le début de la troisième vague (janvier et février) où les tests ont été massifs et assez aléatoires. Par ailleurs, les résultats des tests compilés dans SI-DEP (Système d'information de dépistage populationnel) sont affectés aux lieux de résidence habituels des patients (grâce à la carte Vitale) et non au lieu où sont faits les dépistages, un écart qui peut biaiser les données épidémiologiques régionales en période de congés notamment.

\section{LES EAUX USÉES RECUEILLENT LE VIRUS EXCRÉTÉ DANS LES SELLES DES SUJETS SYMPTOMATIQUES ET NON SYMPTOMA- TIQUES}

Bien que l'infection à SARS-CoV-2 affecte en premier lieu les voies respiratoires, le génome viral est également détecté dans le sang, les urines (de façon assez exceptionnelle) et dans les selles (Peng et al. 2020 ; Holshue et al. 2020) où les charges virales peuvent être transitoirement très élevées (de 102 à 108 copies d'ARN par gramme de selle selon certains auteurs) (Wölfel et al. 2020); les durées d'excrétion dans les selles, selon une dynamique qui reste toujours à préciser, varient d'un patient à l'autre. Elles peuvent s'étendre à 3, 6 voire 17 semaines et peuvent excéder la détection du virus dans les voies respiratoires supérieures (Chen et al. 2020 ; Wölfel et al. 2020 ; Wu et al. 2020 ; Zhang et al. 2021). Ces observations suggèrent qu'il s'agit principalement d'une réplication virale entérique et non du transit passif d'un virus d'origine naso-pharyngée au tube digestif. Selon une méta-analyse publiée en 2020 et réunissant 47 études, les patients atteints de COVID-19 manifestent divers signes d'atteinte gastro-entérique à type de diarrhées (7,7 \%), de nausées/vomissements $(7,8 \%)$ et de douleurs abdominales (2,7\%) (Sultan et al. 2020). Ces signes sont imparfaitement corrélés à la présence du virus dans les selles puisque le génome viral est détecté dans près de $50 \%$ des échantillons de selle prélevés chez les malades et que certaines selles de patients atteints de diarrhée sont négatives (Lin et al. 2020 ; Tian et al. 2020 ; Guo et al. 2021). Il est important de noter que le génome viral peut être détecté chez les adultes et les enfants et notamment chez des porteurs peu ou pas symptomatiques (Jones et al. 2020 ; Xu et al. 2020). En conclusion, la détection du génome viral dans les selles est bien documentée mais les données qui permettraient de caractériser la dynamique de l'excrétion restent parcellaires : les mesures de charges virales chez les patients asymptomatiques sont rares et l'impact de la vaccination sur le cycle entérique du virus et sur son excrétion dans les selles n'est pas solidement documentée à ce jour (Schmitz et al. 2021). Par ailleurs, la possibilité d'une transmission féco-orale du SARS-CoV-2 reste discutée : les preuves épidémiologiques sont assez fragiles et l'isolement de virus infectieux à partir de selles contenant du génome viral reste assez rare (Xiao et al. 2020 ; Zhang et al. 2020 ; Wang et al. 2020 ; Guo et al. 2021).

Dès l'hiver 2020, plusieurs équipes souvent issues de la microbiologie environnementale s'attachent à rechercher la présence du virus dans les eaux usées notamment au niveau des stations de traitement des eaux usées (STEU). Gertjan Medema et ses collaborateurs sont les premiers à publier - aux Pays-Bas que le SARS-CoV-2 peut être détecté dans les eaux usées concomitamment à l'identification des premiers cas cliniques (Medema et al. 2020). L'utilisation d'échantillons d'eaux usées analysés de façon rétrospective permettra même de détecter le virus avant que les premiers cas cliniques ne soient identifiés ou dans les zones de faible prévalence (Ahmed et al. 2020a ;Peccia et al. 2020 ; Randazzo et al. 2020 ; La Rosa et al. 2020). Toutefois, ces approches restent qualitatives. Si elles constituent un signal d'alerte précoce, elles ne permettent pas de caractériser la dynamique de l'épidémie ni, a fortiori, de mesurer l'impact des mesures de freinage collectives (confinement, couvre-feu) ou d'identifier une reprise épidémique à l'échelle d'un territoire. Elles ne permettent pas non plus de construire des modèles épidémiologiques capables de mettre en relation les quantités de virus recueillies dans les eaux usées avec le nombre de porteurs du virus. C'est pour répondre à ces questions qu'un premier consortium de recherche s'est construit dès mars 2020 autour de trois équipes de Sorbonne Université (virologie, mathématiques, hydrologie), de l'équipe R\&D d'Eau de Paris (virologie environnementale) et de l'Institut de recherche biomédicale des armées (IRBA, virologie) afin de quantifier le génome viral dans les STEU. Dans l'étude princeps réalisée sur les STEU d'île-de-France - contemporaine de l'étude hollandaise - le génome viral a été extrait et quantifié par RT-qPCR entre le 5 mars et le 23 avril 2020, une période qui inclut le premier 
confinement (17 mars - 11 mai 2020). Il faut rappeler qu'à cette période, les campagnes de tests RT-qPCR massives n'ont pas encore débuté en France. Par conséquent, l'évaluation de la dynamique de l'épidémie repose principalement sur les données cliniques (cas présomptifs et éventuellement confirmés). Les résultats obtenus démontrent que la mesure hebdomadaire des charges virales dans les eaux usées est très bien corrélée avec le nombre d'hospitalisations ; par ailleurs, on observe une réduction drastique des charges virales une à deux semaines seulement après la mise en place des mesures de confinement en France, réduction qui annonce la diminution du nombre de patients hospitalisés dans les semaines qui suivront (Figure 1).
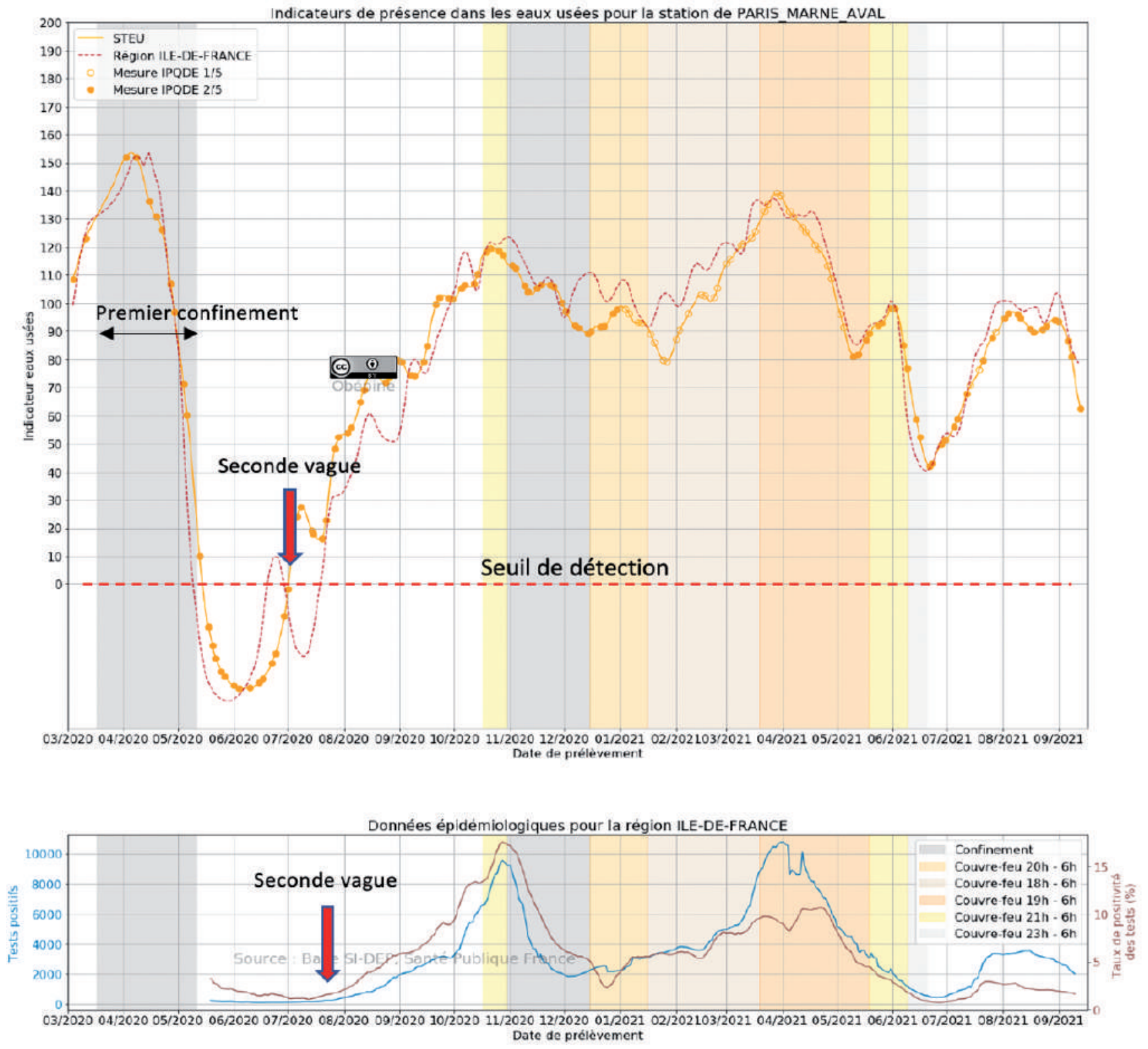

Figure 1 : Dynamique de l'épidémie de COVID-19 en Île-de-France : niveau de circulation dans les eaux usées (vignette supérieure) et dans la population (vignette inférieure)

Haut: Obépine produit un indicateur sans unité, semi-logarithmique, qui traduit le niveau de circulation dans les STEU. Les valeurs moyennes de l'indicateur en Île-de-France (courbe pointillée) et les valeurs mesurées sur la station Marne - Aval (courbe pleine) sont indiquées. Les périodes de confinement apparaissent en gris. Les périodes colorées correspondent à diverses mesures de freinage (couvre-feu).

Bas : Taux d'incidence et taux de positivité en Île-de-France

La flèche rouge indique la détection de la seconde vague dans les eaux usées (20 juin) et dans la population (fin juillet). 
La prépublication de l'article publié sur la région Île-de-France (Wurtzer et al. 2020) - largement relayée dans la presse scientifique (Lesté-Lasserre, 2020) et dans les médias français et étrangers - démontre pour la première fois l'intérêt d'une approche quantitative pour construire un dispositif de suivi de l'épidémie via les eaux usées, notamment dans des situations où les ressources technologiques, humaines et/ou financières sont insuffisantes pour documenter la dynamique de l'épidémie par des campagnes de tests massifs. Des travaux similaires sont conduits à Nancy par le Laboratoire de Chimie Physique et Microbiologie pour les Matériaux et l'Environnement (LCPME) sur 10 des plus grandes villes françaises (Bertrand et al. 2021), une équipe qui participera également à la construction du consortium de recherche Obépine (voir infra). En dépit d'une certaine réticence de la communauté médicale française qui a parfois opposé l'épidémiologie des eaux usées aux approches «indivi du-centrées», cette stratégie a été soutenue par trois académies (technologies, sciences et médecine) avant d'être identifiée comme un axe de recherche prioritaire par le Comité Analyse, Recherche et Expertise (CARE) COVID-19 présidé par Françoise Barré-Sinoussi. Ces soutiens ont permis, dès mai 2020, d'élargir le consortium initial pour créer l'Observatoire épidémiologique dans les eaux usées (Obépine), un dispositif de recherche intégré (virologie humaine, mathématiques, hydrologie, virologie environnementale, évaluation du risque virologique des mollusques d'eau douce et d'eau de mer) qui évoluera en août 2020 pour devenir un groupement d'intérêt scientifique (GIS) ${ }^{1}$. Les problématiques proposées abordent tous les aspects de l'épidémiologie du SARS-CoV-2 via les eaux usées, et au-delà de répondre aux questionnements scientifiques suivants :

- Quels sont les risques infectieux liés aux selles des patients, au virus détecté en amont des stations, dans les boues résiduaires et dans les eaux de surface?

- Quels sont les dispositifs les plus adaptés pour une surveillance environnementale locale (sorties d'eaux usées des bâtiments à usage collectif, écoles, EHPAD, prisons, campus universitaires...)? - Quelles sont les meilleures méthodes de prélèvement et d'analyse? Quels sont les dispositifs de capture du virus les plus efficaces (échantillons bruts ou traités, bio-concentrateurs animaux, capteurs passifs...), les meilleurs sites d'échantillonnage? - Comment construire - à partir des données brutes (charges virales journalières mesurées dans les STEU) - un indicateur fiable, insensible aux précipitations, comparable d'un réseau d'assainissement à l'autre?

- Comment intégrer les paramètres qui influencent les données brutes (densité des populations, gestion des réseaux d'eaux usées, pluviométrie, composition chimique etc.) pour normaliser les signaux au plus près des variations tendancielles réelles de l'épidémie?
- Comment exploiter les techniques de biologie moléculaire et de métagénomique pour analyser la viabilité du virus et la variabilité génétique des virus collectés dans des matrices environnementales?

En juin 2020, le Ministère français de l'Enseignement supérieur, de la Recherche et de l'Innovation (MESRI) a missionné les porteurs du projet pour mettre en place un dispositif sentinelle pilote qui devait produire une carte dynamique hebdomadaire de l'épidémie sur le territoire national métropolitain et outre-mer. Initialement conçu pour prendre en charge 150 stations de référence et doté d'un budget de 3,5 millions d'euros, ce dispositif intègre aujourd'hui 200 STEU et produit chaque semaine une photographie de l'épidémie qui englobe plus de 22 millions de français, soit $33 \%$ de la population, pour un coût 2500 fois inférieur (!) à celui des courbes épidémiologiques construites à partir des données SI-DEP (environ 75000 euros pour un suivi bihebdomadaire de l'ensemble des stations).

\section{D'UNE PREUVE DE CONCEPT À LA CONSTRUCTION D'UN RÉSEAU SENTINELLE NATIONAL}

Le choix des 150 STEU sentinelles initiales du projet (dites stations mères) à partir des 22000 STEU françaises s'est appuyé sur un travail préliminaire de statistique (non publié). Une trentaine de critères hydrologiques, démographiques, économiques, sociologiques, touristiques ont été pris en compte pour établir leur classification en aires urbaines (Tableau I).

1. La somme des populations municipales des communes présentes dans l'aire urbaine ;

2. Le nombre de stations touristiques désignées par décret ministériel au sein de l'aire urbaine ;

3. Le nombre de communes présentes dans l'aire urbaine ;

4. La proportion d'habitants présents dans l'aire urbaine par rapport à celle du département ;

5. La proportion de population active sur la population totale de l'aire urbaine ;

6. La proportion de population retraitée sur la population totale de l'aire urbaine ;

7. Le nombre de supermarchés ;

8. Le nombre de restaurants ;

9. Le nombre d'établissements de divertissement en intérieur (cinémas, théâtres...) ;

10. Le nombre de types d'équipements sportifs présentant un risque de contact élevé entre pratiquants (gymnases, centre-équestres, piscines...) ;

11. Le nombre d'hôtels et de campings (vient compléter le label stations touristiques pour les villes n'étant pas labellisées mais

1- Membres fondateurs du GIS : Sorbonne Université, CNRS, Inserm (Laboratoire Jacques Louis Lions (LJLL) UMR 7598 ; Yvon Maday / Milieux environnementaux, transferts et interactions dans les hydrosystèmes et les sols (METIS) UMR 7619 ; Jean-Marie Mouchel / Centre de Recherche Saint-Antoine (CRSA) UMR S 938 ; Vincent Maréchal) : Université de Lorraine, CNRS (Laboratoire de Chimie Physique et Microbiologie pour les Matériaux et l'Environnement (LCPME) UMR 7564 ; Christophe Gantzer, Isabelle Bertrand) - Eau de Paris (Laboratoire de Recherche et Développement (LR\&D) ; Laurent Moulin, Sébastien Wurtzer) - Service de santé des armées (Institut de recherche biomédicale des armées (IRBA) (Rémy Teyssou, Mickaël Boni)) - IFREMER (Laboratoire Santé Environnement et Microbiologie (LSEM), Soizick Le Guyader) - Université Clermont-Ferrand Auvergne (Laboratoire Microorganismes : Génome et Environnement (LMGE) UMR 6023 ; Jean-Luc Bailly) 
présentant malgré tout un nombre d'équipements touristiques important) ;

12. Le nombre de voyageurs annuels en gare SNCF;

13. Le nombre de voyageurs annuels en aéroport;

14. La moyenne des ratios entre capacité en équivalent habitant et population des communes raccordées des stations d'épuration de l'aire urbaine ;

15. Le nombre de stations balnéaires recensées dans l'aire urbaine ;

16. Une variable booléenne permettant de séparer les aires urbaines dont l'altitude minimale est de $700 \mathrm{~m}$ des autres.

17. Le salaire médian des habitants.

18. Le taux de pauvreté.

19. La proportion de résidences secondaires.

20. La proportion de maisons.

21. Le nombre de préfectures et sous-préfectures dans une aire urbaine.

22. Le nombre de collèges / lycées ;

23. Le nombre d'établissements d'enseignement supérieur ;

24. La taille de flux de travailleurs / étudiants qui résident en dehors de la commune ;

25. La taille de flux de travailleurs / étudiants qui travaillent / étudient en dehors de la commune.

Tableau I : Critères utilisés pour la stratification des 22000 stations de traitement des eaux usées françaises. D'autres éléments non listés (ex: éléments techniques concernant la station et le réseau sanitaire de la collectivité) ont également été pris en compte.

Les stations retenues visent à donner une image de la France allant des métropoles aux communes rurales (Figure 2).

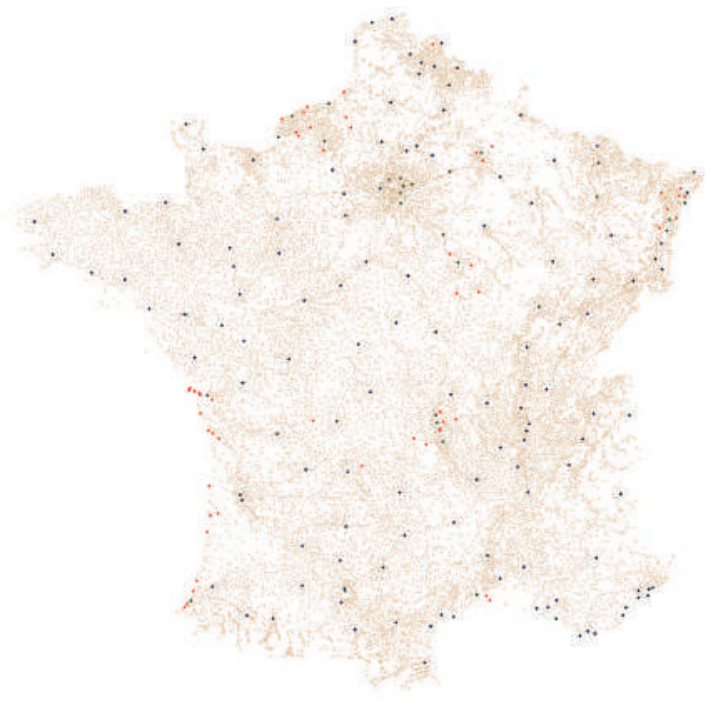

Figure 2 : Localisation des STEU suivies par Obépine en France métropolitaine. Obépine suit actuellement 200 STEU en France métropolitaine (carte) et outre-mer (non représentées ici). Ces 200 stations ont pour l'essentiel été choisies à partir des 22000 STEU françaises sur une stratification statistique incluant près de 30 paramètres (tableau I).
À chaque station mère ont été associées 10 stations filles, de mêmes caractéristiques mais de localisation géographique distincte. Ces stations filles ont été pré-positionnées afin de pouvoir analyser, si nécessaire, les spécificités d'une tendance épidémique locale (zone locale de couvre-feu par exemple). Les opérateurs publics et privés (Suez, VEOLIA, SAUR, SIAAP) ont souhaité s'intégrer au projet en mobilisant les STEU dont ils assuraient le suivi, une mise en contact qui s'est assortie de la signature d'une charte précisant à la fois les conditions du suivi (coûts portés par la dotation ministérielle reçue par Obépine) et la mise à disposition des données aux autorités de santé et aux collectivités.

\section{Recrutement des laboratoires : les protocoles, les essais inter-laboratoires (EIL), la collecte des données}

Les équipes d'Obépine ont produit plusieurs protocoles expérimentaux, précisant à la fois les conditions de prélèvements et de conservation et les méthodes analytiques. Ces protocoles de même que leurs versions optimisées ont été diffusés, sous réserve qu'aucun usage commercial n'en soit fait, à tout laboratoire désirant participer à la production des quelques 400 analyses hebdomadaires (deux prélèvements hebdomadaires sur 200 STEU). La plupart des analyses ont été conduites par RT-PCR quantitative (RT-qPCR), exceptionnellement par PCR digitale (dPCR). Des essais inter-laboratoires réguliers ont permis d'identifier certaines difficultés opérationnelles majeures :

- disponibilité parfois limitée de certains équipements, matériels et réactifs pour beaucoup communs aux analyses faites chez l'homme, ce qui a parfois conduit à des difficultés d'approvisionnement en période de crise ;

- expertise en biologie moléculaire minimale indispensable pour mettre en ouvre et adapter des protocoles issus de la recherche, requérant parfois des formations personnalisées ; - absence de "standards " pour valider les techniques de RT-qPCR/dPCR, et les calibrer, qu'il s'agisse d'échantillons naturels ou synthétiques, du choix des régions du génome viral à amplifier, des techniques d'extraction ou d'amplification, voire même de critères d'analyse ;

- difficulté à normaliser les données de charges virales brutes : présence d'inhibiteurs nombreux et/ou mal identifiés, absence de standard consensuel pour normaliser les charges virales aux tailles des populations.

Les laboratoires auxquels les analyses ont été confiées (11 actuellement, dont des laboratoires vétérinaires) ont été retenus à l'issue de plusieurs campagnes d'essais inter-laboratoires qui ont été initiées dès septembre 2020. Il est évident, avec le recul, que cette phase du dispositif, avec des moyens humains plus importants, aurait sans doute requis des ateliers de formation et/ou d'échanges de pratique qui n'ont pu être systématiquement proposés aux laboratoires qui rencontraient des difficultés. La publication par l'Union 
Européenne $^{2}$ de recommandations préconisant de déployer, dans chaque état membre, un dispositif proche de celui mis en place par Obépine a sans aucun doute contribué à promouvoir - un peu tardivement - la création de groupes de normalisation, notamment à l'AFNOR, et à motiver une prise en charge officielle du réseau de surveillance par les services de l'état (projet SUM'EAU, porté par la direction générale de la santé et la direction de l'eau et de la biodiversité).

\section{Expression des résultats : des données brutes à la construction d'un indicateur de suivi du virus dans les eaux usées}

L'exploitation épidémiologique des données de charge virale dans les eaux usées est assujettie à la construction de courbes de tendance reflétant les variations des quantités journalières de virus mesurées en entrée de STEU (Figure 1). Les données brutes (exprimées en équivalents génome / L) produites par les laboratoires sur une base bihebdomadaire sont par essence bruitées. L'utilisation de prélèvements moyennés sur $24 \mathrm{~h}$ permet de lisser les variations de charge virale au sein d'une même journée. Nous avons pu démontrer que deux (idéalement trois) prélèvements hebdomadaires sont nécessaires et suffisants pour produire des courbes de tendance fiables (Cluzel et al. 2021). Toutefois, de nombreux autres facteurs de variabilité intra- et inter-expérimentaux, liés notamment aux techniques de mesure en milieu biologique, à la composition chimique des eaux usées, à leur dilution éventuelle, génèrent un bruit qu'il est nécessaire d'intégrer et de corriger par l'utilisation d'algorithmes mathématiques prenant en compte la modélisation de l'épidémie et sa traduction dans la présence du virus dans les selles des personnes infectées et donc dans les eaux usées, notamment si l'on souhaite pouvoir comparer les courbes d'un territoire à l'autre ou interpréter les changements de tendance (Arabzadeh et al. 2021 ; Bivins et al. 2021). Afin de répondre à cette contrainte, les équipes d'Obépine ont construit un filtre mathématique dit de Kalman, proposant de traiter les données temporelles comme une variable ayant une dynamique et bruitée par des erreurs. Ce filtre permet de lisser ces erreurs et les courbes proposées s'améliorent au fil de l'acquisition des données. Outre les erreurs sur les mesures, l'algorithme proposé permet de reconstruire des courbes tendancielles sous les limites de détection ou en écartant les données visiblement aberrantes (Courbariaux et al. 2021). Ce lisseur est parfaitement adapté pour reconstruire des courbes de tendance estimées au plus près des courbes réelles (Figure 3). Les très nombreuses données acquises ces derniers mois incitent même à penser que ce modèle pourrait devenir prédictif : à l'heure actuelle, il est en effet possible de prédire les évolutions de charge virale dans les eaux usées à 7 jours avec une fiabilité supérieure à $70 \%$ (Cluzel et al. en préparation), un élément déterminant pour mettre en place des plans d'action au plus près des évolutions locales de l'épidémie.
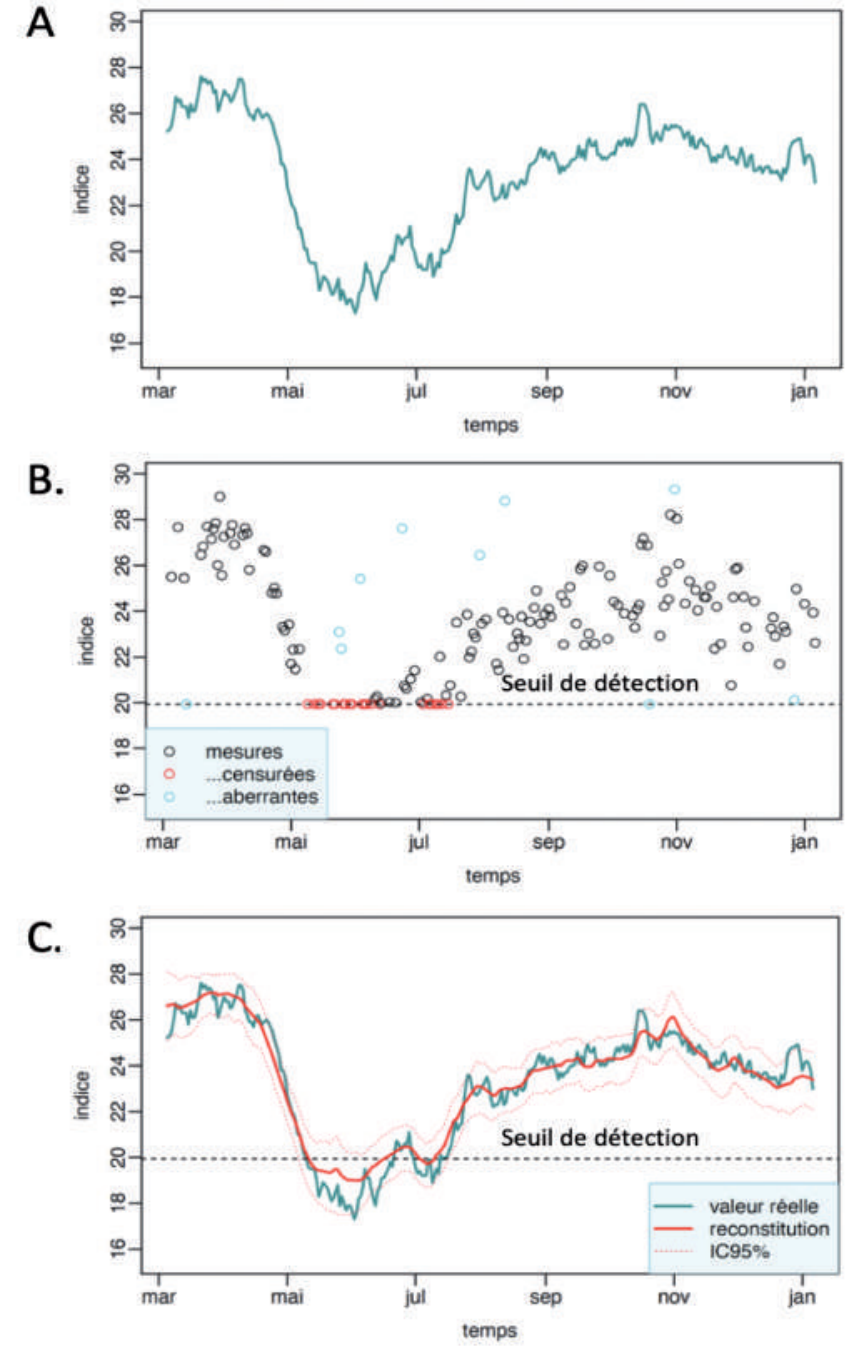

Figure 3 : Filtre mathématique utilisé pour lisser les données de l'indicateur eaux usées. Un indicateur sans unité (Indicateur Eaux Usées) a été généré à partir des données de charge virale obtenues pour chaque STEU. Les données étant par essence bruitée, Obépine a conçu un filtre mathématique (Courbariaux et al. 2021) permettant de lisser les valeurs de cet indicateur et de comparer l'évolution de la circulation virale d'une station à l'autre. Ce filtre vise à reconstituer les données, à partir des données expérimentales, au plus près des valeurs réelles (inconnues par définition).

La figure a été conçue à partir d'une courbe modèle (courbe verte, vignette A). Des pseudo-données expérimentales ont été générées à partir de la courbe réelle (vignette $B$ ). Les valeurs générées sont variables, contiennent des valeurs aberrantes (cercles bleus) et des valeurs inférieures au seuil de détection (cercles rouges). La vignette $\mathrm{C}$ montre que l'application du lisseur mathématique aux données expérimentales permet de reconstituer une courbe de tendance estimée à partir des pseudo-données expérimentales (courbe rouge) très proche des valeurs réelles (courbe verte).

Les STEU intégrées aux réseaux présentent des caractéristiques propres (dont la capacité de traitement, les variations d'activité, la composition des effluents) qui rendent complexe la comparaison des données obtenues entre différentes STEU. 
Nous avons fait le choix de construire - à partir des données des moins coûteux) pour suivre les changements de tendance de brutes (Eq génomes/L) un indicateur sans unité (indicateur eaux l'épidémie, notamment à l'échelle d'un bassin versant.

usées ou IEU) de circulation du virus dans les eaux usées : cet indicateur semi-logarithmique, construit à partir des concentrations de génome viral, est au suivi du SARS-CoV-2 dans les eaux usées ce que l'échelle de Richter est à l'intensité des secousses sismiques (Cluzel et al. 2021). L'IEU, dès lors qu'il intègre les fluctuations de débit des stations, montre une excellente robustesse même lors des épisodes de fortes précipitations. À l'occasion des deuxièmes et troisièmes vagues de COVID-19, nous avons été en mesure de comparer les changements de tendance de l'IEU avec les données épidémiologiques locales. Ces travaux confirment d'abord qu'il existe en général une excellente corrélation entre les variations de l'IEU et celles des taux d'incidence au niveau de la collectivité et au niveau régional ; par ailleurs, si les variations de l'IEU précèdent de 3 jours en moyenne les tendances observées sur les taux d'incidence, cette avance peut exceptionnellement atteindre deux semaines (Cluzel et al. 2021). Cette capacité à anticiper les reprises épidémiques à travers le suivi des eaux usées confirme les observations qui avaient conduit Obépine à signaler le retour du virus en Île-de-France tout comme dans d'autres villes françaises (e.g. Nancy) dès le début de l'été 2020, soit près d'un mois avant que d'autres signaux épidémiologiques ne confirment le début de la

\section{La production des données et la mise à disposition des autorités sanitaires et du grand public}

Les données produites par les laboratoires du réseau Obépine sont produites avec un financement du MESRI. Elles s'inscrivent de fait dans le champ de la loi 2016-1321 du 7 octobre 2016 pour une République numérique (dite «loi Lemaire») en faveur de la "circulation des données et du savoir». La mise à disposition des données produites par les laboratoires a été envisagée à trois niveaux au moins : les autorités sanitaires, afin de guider éventuellement des plans d'action régionaux, les collectivités qui ont accepté d'intégrer leur STEU au réseau de surveillance et le grand public. Un serveur sécurisé dédié a été construit afin d'assurer le transfert en temps réel des données brutes produites par les laboratoires. Converties en IEU, les données sont secondairement déposées sur la plateforme gouvernementale dédiée (https://covid-19.sante.gouv.fr/) afin d'assurer la transmission aux acteurs de santé. Parallèlement, Obépine adresse chaque semaine aux ARS et aux collectivités un rapport détaillé sur la situation de chacune des stations suivies. Suivant un bref embargo (72 h), ces données sont mises en ligne à destination du grand public sur le site du réseau seconde vague (Wurtzer et al. soumis à publication). Ces résultats (https://www.reseau-obepine.fr) où elles sont associées à une
confirment que la surveillance du génome viral dans les eaux carte nationale des tendances sur 7 (Figure 4 ) et 30 jours (non usées proposée reste l'un des indicateurs les plus avancés (et l'un montré) (https://www.reseau-obepine.fr/carte-des-tendances/).

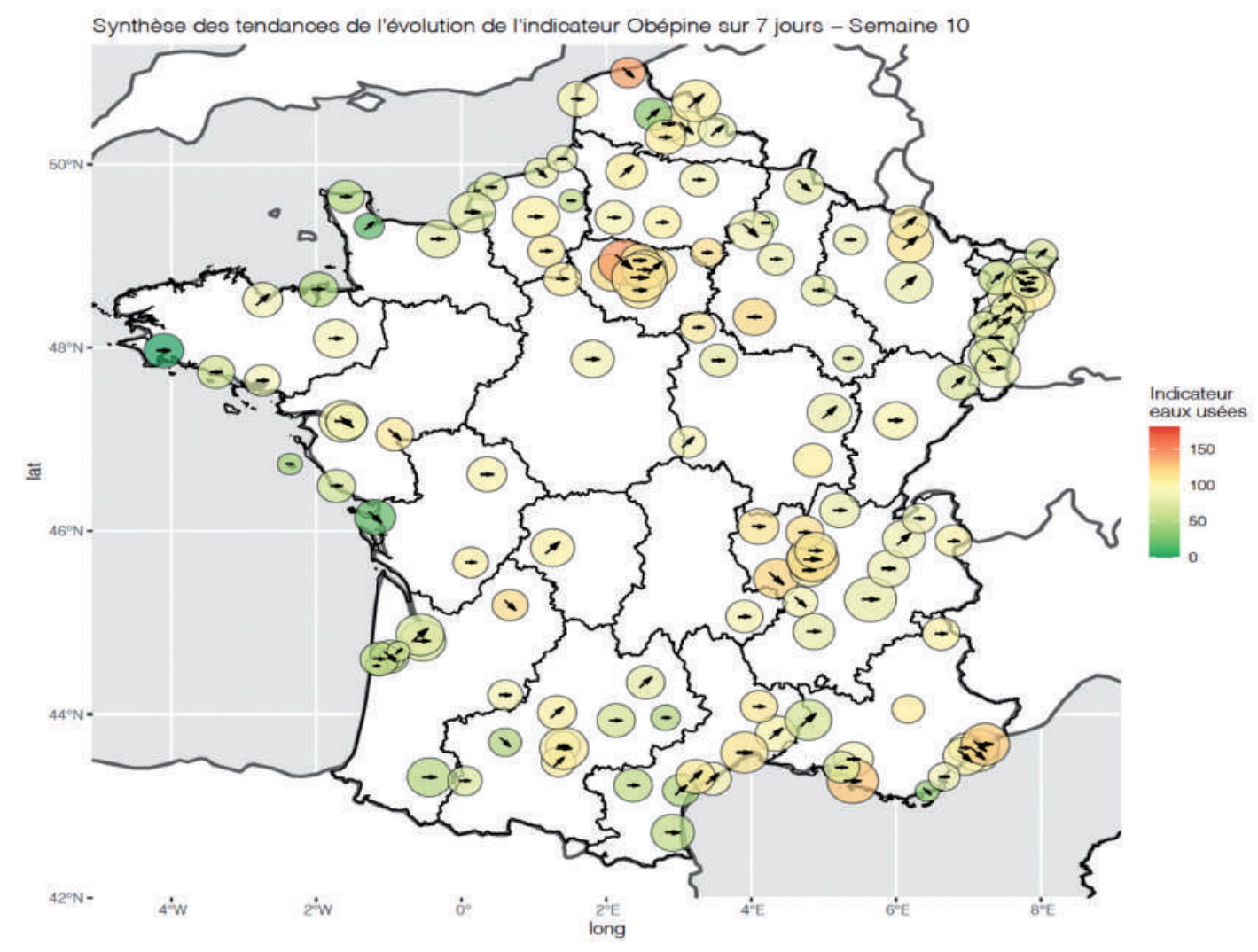

Figure 4: Carte tendancielle de la circulation du SARS-CoV-2 via les eaux usées. Le réseau Obépine produit chaque semaine une carte indiquant les tendances de circulation virale sur le territoire national à 7 jours (ici) et 30 jours (non montré). Exemple : la semaine 10 de l'année 2021. 


\section{ÉPIDÉMIOLOGIE DES EAUX USÉES : PREMIER RETOUR D'EXPÉRIENCE}

Le consortium de recherche Obépine a permis, en l'espace de quelques mois seulement, de proposer et de mettre en place un réseau de surveillance national de l'épidémie de COVID-19. Des initiatives similaires ont été mises en place à travers le monde, démontrant de façon inédite à cette échelle le potentiel de l'épidémiologie des eaux usées dans la prévention et le suivi d'une épidémie (Bibby et al. 2021 ; Kitajima et al. 2020).

Appuyé sur un réseau actuel de 200 stations, mais incluant également un suivi ciblé au niveau de collecteurs parisiens, Obépine tire son efficacité d'une approche transdisciplinaire qui a réuni des spécialistes de la virologie médicale et environnementale, des microbiologistes, des mathématiciens, des hydrologues soucieux de combiner une recherche académique de haut niveau et d'en transférer les acquis pour proposer de nouveaux outils d'aide à la décision. Cette double ambition a parfois généré une certaine confusion entre les activités de recherche et de surveillance du consortium. Les opérateurs en charge des stations, sollicités pour déployer le réseau, ont joué un rôle important quoique parfois ambivalent comme en témoigne l'existence d'offres commerciales qui ont émergé en marge du projet de collaboration initial. Ces difficultés sont sans doute inhérentes à la construction d'un projet qui a grandi très vite, motivé par l'importance sanitaire qu'il portait, mais hors d'un cadre partenarial clair. La constitution d'un réseau pérenne de laboratoires experts reste également un objectif important. En l'absence de norme internationale, les analyses ont été conduites à partir de protocoles de recherche dont l'exécution - parfois complexe - a été validée à travers des essais inter laboratoires organisés par un laboratoire expert de l'IFREMER avec l'objectif de produire un indicateur pertinent, ce qui a conduit à être particulièrement vigilant sur la sensibilité et la reproductibilité des mesures. Des expériences identiques, notamment aux Pays-Bas, ont été facilitées par une centralisation plus claire du portage du projet, confié officiellement à un seul laboratoire expert apte à développer de concert recherche fondamentale et surveillance à l'échelle nationale. Le portage du projet, en France, a sans doute été complexifié par l'absence d'un pilotage à même de réunir de façon simple des acteurs de compétence variées, réunis pour traiter d'un sujet commun relevant de la santé globale. Le portage du projet par le MESRI, puis son transfert à une structure mixte (SUM'EAU) portée par le Ministère de la Solidarité et de la Santé d'une part et le Ministère de la Transition Écologique d'autre part témoigne d'une difficulté à inscrire les projets de santé globale dans une structure alliant recherche académique et déploiement opérationnel. En effet, le consortium de recherche ou GIS Obépine n'est pas qu'un réseau de surveillance. Les équipes qui le composent balaient de nombreuses questions fondamentales et appliquées portant sur l'infectiosité du virus dans les selles (Wurtzer et al. en préparation), l'intégrité des particules virales dans les eaux usées (Wurtzer et al. 2021b ; Wurtzer et al. en préparation), la persistance du virus dans les boues (Varbanov et al. 2021) ou dans les coquillages (Desdouits et al. 2021) ou bien encore sur l'impact des traitements des boues et des eaux usées (Serra-Compte et al. 2021). Une étude récente conduite sur le porte-avions Charles de Gaulle (Gorgé et al. en préparation) confirme l'intérêt d'un suivi des eaux noires, une stratégie qui a également été mise à profit pour la surveillance des vols internationaux (Ahmed et al. 2020b).

Les eaux usées permettent de suivre les formes classiques du virus, qui ont prédominé en France jusqu'en décembre 2020, mais également de certains variants. Ainsi, le suivi mené en Île-de-France démontre que le variant alpha, identifié dès le 4 janvier 2021 dans les STEU parisiennes, a progressivement supplanté les formes antérieures du virus (Wurtzer et al. soumis). L'émergence de variants viraux de contagiosité accrue, susceptibles d'échapper à la réponse immunitaire et/ou plus virulent pose de nouveaux défis. Si le suivi des variants dans les eaux usées ouvre des perspectives intéressantes en matière de recherche fondamentale - notamment parce que les approches génétiques et métagénomiques pourraient être utilisées pour d'autres microorganismes - il conviendra de s'interroger sur la nécessité de suivre les variants viraux dans les eaux usées dans le cadre d'un plan de surveillance généralisé et de la méthode à utiliser (recherche ciblée des mutations par RT-PCR, séquençage ciblé ou approche méta génomique).

Les épidémies récentes qui ont touché les élevages de visons aux Pays-Bas et au Danemark (Elaswad et al. 2020 ; Oude Munnink et al. 2021) nous rappellent que le SARS-CoV-2, issu du monde animal, pourrait bien y trouver de nouveaux hôtes (Goraichuk et al. 2021). Surveiller l'émergence du SARS-CoV-2 dans les espèces animales et a fortiori chez celles qui côtoient l'Homme (animaux de compagnie ou d'élevages, rongeurs urbains) est essentiel pour éviter que le virus ne s'installe dans de nouveaux réservoirs - ce qui en compliquerait notoirement le contrôle ou bien encore que ne soient sélectionnés de nouveaux variants, à l'instar de ce qui a été observé chez le vison d'élevage. Un dispositif de surveillance des eaux usées, des effluents agricoles, ou l'analyse des selles des animaux domestiques ou sauvages - incluant les rongeurs urbains - pourrait sans aucun doute enrichir un dispositif intégré de surveillance de l'épidémie. Obépine et la plupart des projets similaires qui ont été mis en place en Europe et ailleurs, mettent en avant une approche épidémiologique peu coûteuse, et donc particulièrement bien adaptée aux pays à faible ressources économiques. Il n'est pas inutile de rappeler que le suivi épidémiologique proposé par Obépine coûte environ 75000 euros par semaine et couvre plus de $33 \%$ de la population française ; d'un autre côté, certaines semaines, plus de 200 millions d'euros ont été dépensés en tests individuels pour suivre une part bien plus faible de la population française. Même si la vocation des tests individuels est différente, ces coûts peuvent et doivent être mis en regard. À l'heure où le déremboursement des tests dits " de confort " est programmé, le suivi des eaux usées pourrait s'imposer comme une stratégie alternative de choix, homogène dans le temps et dans les territoires, pour maintenir une surveillance épidémiologique de qualité pour un coût supportable. Le transfert des activités de surveillance portées par Obépine vers le dispositif SUM'EAU devrait donc veiller à ne pas interrompre un dispositif 
de surveillance opérationnel à une période critique de l'épidémie. Plus largement, l'analyse des eaux usées - avec des adaptations techniques et stratégiques judicieuses notamment pour faciliter l'analyse de prélèvements ponctuels à l'aide de capteurs adaptés (Hayes et al. 2021) dans les pays qui ne disposent pas d'un réseau d'assainissement suffisamment structuré - pourrait aussi répondre aux besoins sanitaires de pays durement touchés par l'épidémie qui n'ont pas les ressources humaines, techniques, économiques pour suivre les épidémies, voire les épizooties à travers des campagnes massives de tests individuels réalisés sur les humains ou les animaux.

\section{REMERCIEMENTS}

Le projet de recherche Obépine a reçu des soutiens financiers du CNRS, de Sorbonne Université et du Ministère de l'Enseignement Supérieur, le Recherche et de l'Innovation et d'Eau de Paris. Les auteurs sont reconnaissants aux opérateurs (SAUR, Suez, Véolia, SIAAP) qui ont facilité la collecte des échantillons, aux collectivités qui ont accompagné le projet et aux laboratoires qui ont assuré des analyses (Ifremer Nantes, Inovalys, CIRSEE, LDA76, Qualyse, IUT Louis Pasteur, Eau de Paris, HSM, LGME, Actalia, LCPME, GEIST Limoges). Ils souhaitent également remercier Dominique Vernhet, Olivia Leroy, Iris Kerambrun et Cécile Arenes pour leur contribution au pilotage, à la construction et à l'animation du projet, ainsi que les nombreux collègues qui ont produit et/ou analysé les données, ont construit et validé les modèles: Prunelle Waldman, Eloise Dhenain, Morgane Levert, Elise Richard, Chloé Borde, Nicolas Cluzel, Marie Courbariaux, Siyun Wang, Karine Laurent.

\section{CONFLITS D'INTERETS}

Les auteurs déclarent n'avoir aucun conflit d'intérêt.

\section{BIBLIOGRAPHIE}

- Ahmed W, Angel N, Edson J, Bibby K, Bivins A, O'Brien JW, et al. First confirmed detection of SARS-CoV-2 in untreated wastewater in Australia: A proof of concept for the wastewater surveillance of COVID-19 in the community. Science of The Total Environment 2020a; 728:138764.

- Ahmed W, Bertsch PM, Angel N, Bibby K, Bivins A, Dierens L, et al. Detection of SARS-CoV-2 RNA in commercial passenger aircraft and cruise ship wastewater: a surveillance tool for assessing the presence of COVID-19 infected travellers. Journal of Travel Medicine 2020b;27:taaa116.

- Arabzadeh R, Gruenbacher DM, Insam $\mathrm{H}$, Kreuzinger N, Markt R, Rauch W: Data filtering methods for SARS-CoV-2 wastewater surveillance (Internet). arXiv:210411566 (q-bio, stat) 2021 (cited 2021 Sep 2);Available from: http://arxiv.org/abs/2104.11566

- Bertrand I, Challant J, Jeulin H, Hartard C, Mathieu L, Lopez S, et al. Epidemiological surveillance of SARS-CoV-2 by genome quantification in wastewater applied to a city in the northeast of France: Comparison of ultrafiltrationand protein precipitation-based methods.
International Journal of Hygiene and Environmental Health 2021;233:113692. - Bibby K, Bivins A, Wu Z, North D: Making waves: Plausible lead time for wastewater based epidemiology as an early warning system for COVID-19. Water Research 2021;202:117438.

- Bivins A, Kaya D, Bibby K, Simpson SL, Bustin SA, Shanks OC, et al. Variability in RT-qPCR assay parameters indicates unreliable SARS-CoV-2 RNA quantification for wastewater surveillance. Water Research 2021;203:117516.

- Chen C, Gao G, Xu Y, Pu L, Wang Q, Wang L, et al. SARS-CoV-2-Positive Sputum and Feces After Conversion of Pharyngeal Samples in Patients With COVID-19. Ann Intern Med 2020; DOI: 10.7326/M20-0991

- Cluzel N, Courbariaux M, Wang S, Moulin L, Wurtzer S, Bertrand I, et al. Mathematical modeling and adequate environmental sampling plans are essential for the public health assessment of COVID-19 pandemics: development of a monitoring indicator for SARS-CoV-2 in wastewater (Internet), Epidemiology, 2021. DOI: 10.1101/2021.09.01.21262877 - Corman VM, Muth D, Niemeyer D,
Drosten C: Hosts and Sources of Endemic Human Coronaviruses (Internet); in : Advances in Virus Research. Elsevier, 2018, pp 163-188.

- Corpuz MVA, Buonerba A, Vigliotta G, Zarra T, Ballesteros F, Campiglia P, et al. Viruses in wastewater: occurrence, abundance and detection methods. Science of The Total Environment 2020;745:140910.

Courbariaux M, Cluzel N, Wang S, Maréchal V, Moulin L, Wurtzer S, et al. An autoregressive model for a censored data denoising method robust to outliers with application to the Ob\'epine SARS-Cov-2 monitoring (Internet). arXiv:210802115 (stat) 2021 (cited 2021 Aug 29); Available from: http://arxiv.org/abs/2108.02115

- Danchin A, Ng TW, Turinici G: A New Transmission Route for the Propagation of the SARS-CoV-2 Coronavirus. Biology 2020;10:10.

- Desdouits M, Piquet J-C, Wacrenier C, Le Mennec C, Parnaudeau S, Jousse S, et al. Can shellfish be used to monitor SARS-CoV-2 in the coastal environment? Science of The Total Environment 2021;778:146270. 
- Devault DA, Karolak S: Wastewater-based epidemiology approach to assess population exposure to pesticides: a review of a pesticide pharmacokinetic dataset. Environ Sci Pollut Res 2020;27:4695-4702.

- Devault DA, Maguet H, Merle S, Péné-Annette A, Lévi Y: Wastewater-based epidemiology in low Human Development Index states: bias in consumption monitoring of illicit drugs. Environ Sci Pollut Res 2018;25:27819-27838.

- Di Marco M, Baker ML, Daszak P, De Barro P, Eskew EA, Godde CM, et al. Opinion: Sustainable development must account for pandemic risk. Proc Natl Acad Sci USA 2020;117:3888-3892.

- Elaswad A, Fawzy M, Basiouni S, Shehata AA: Mutational spectra of SARS-CoV-2 isolated from animals. Peer] 2020;8:e10609.

- Elsamadony M, Fujii M, Miura T, Watanabe $\mathrm{T}$ : Possible transmission of viruses from contaminated human feces and sewage: Implications for SARS-CoV-2. Sci Total Environ 2021;755:142575.

- Gao Z, Xu Y, Sun C, Wang X, Guo Y, Qiu $\mathrm{S}$, et al. A systematic review of asymptomatic infections with COVID-19. Journal of Microbiology, Immunology and Infection 2021;54:12-16.

- Goraichuk IV, Arefiev V, Stegniy BT, Gerilovych AP: Zoonotic and Reverse Zoonotic Transmissibility of SARS-CoV-2. Virus Research 2021;302:198473.

- Guo M, Tao W, Flavell RA, Zhu S: Potential intestinal infection and faecal-oral transmission of SARS-CoV-2. Nat Rev Gastroenterol Hepatol 2021;18:269-283.

- Hayes EK, Sweeney CL, Anderson LE, Li B, Erjavec GB, Gouthro MT, et al. A novel passive sampling approach for SARS-CoV-2 in wastewater in a Canadian province with low prevalence of COVID-19. Environ Sci: Water Res Technol 2021;7:1576-1586.

- Holshue ML, DeBolt C, Lindquist S, Lofy $\mathrm{KH}$, Wiesman J, Bruce H, et al. First Case of 2019 Novel Coronavirus in the United States. N Engl J Med 2020;382:929-936.

- Hu B, Guo H, Zhou P, Shi Z-L: Characteristics of SARS-CoV-2 and COVID-19. Nat Rev Microbiol 2021;19:141-154.

- Ioannidis JPA: Infection fatality rate of
COVID-19 inferred from seroprevalence data. Bull World Health Organ 2021;99:19-33F.

- Johansson MA, Quandelacy TM, Kada S, Prasad PV, Steele M, Brooks JT, et al. SARS-CoV-2 Transmission From People Without COVID-19 Symptoms. JAMA Netw Open 2021;4:e2035057.

- Jones DL, Baluja MQ, Graham DW, Corbishley A, McDonald JE, Malham SK, et al. Shedding of SARS-CoV-2 in feces and urine and its potential role in person-to-person transmission and the environment-based spread of COVID-19. Science of The Total Environment 2020;749:141364.

- Jones KE, Patel NG, Levy MA, Storeygard A, Balk D, Gittleman JL, et al. Global trends in emerging infectious diseases. Nature 2008;451:990-993.

- Kang M, Wei J, Yuan J, Guo J, Zhang Y, Hang J, et al. Probable Evidence of Fecal Aerosol Transmission of SARS-CoV-2 in a High-Rise Building. Ann Intern Med 2020;173:974-980.

- Kitajima M, Ahmed W, Bibby K, Carducci A, Gerba CP, Hamilton KA, et al. SARS-CoV-2 in wastewater: State of the knowledge and research needs. Science of The Total Environment 2020;739:139076. - La Rosa G, Iaconelli M, Mancini P, Bonanno Ferraro G, Veneri C, Bonadonna $\mathrm{L}$, et al. First detection of SARS-CoV-2 in untreated wastewaters in Italy. Science of The Total Environment 2020;736:139652.

- Le Guyader FS, Ollivier J, Le Saux J-C, Garry P: Les virus entériques humains et l'eau. Revue Francophone des Laboratoires 2014;2014:41-49.

- Lesté-Lasserre C: Coronavirus found in Paris sewage points to early warning system. Science 2020; DOI: 10.1126/science.abc3799

- Lin L, Jiang X, Zhang Z, Huang S, Zhang $Z$, Fang Z, et al. Gastrointestinal symptoms of 95 cases with SARS-CoV-2 infection. Gut 2020;69:997-1001.

- Lorenzo M, Picó Y: Wastewater-based epidemiology: current status and future prospects. Current Opinion in Environmental Science \& Health 2019;9:77-84.

- Mao K, Zhang H, Pan Y, Yang Z: Biosensors for wastewater-based epidemiology for monitoring public health. Water Research 2021;191:116787.

- Mao K, Zhang K, Du W, Ali W, Feng X, Zhang $\mathrm{H}$ : The potential of wastewater-based epidemiology as surveillance and early warning of infectious disease outbreaks. Current Opinion in Environmental Science \& Health 2020;17:1-7.

- McDermott CV, Alicic RZ, Harden N, Cox EJ, Scanlan JM: Put a lid on it: are faecal bio-aerosols a route of transmission for SARS-CoV-2? Journal of Hospital Infection 2020;105:397-398.

- Medema G, Heijnen L, Elsinga G, Italiaander R, Brouwer A: Presence of SARS-Coronavirus-2 RNA in Sewage and Correlation with Reported COVID-19 Prevalence in the Early Stage of the Epidemic in The Netherlands. Environ Sci Technol Lett 2020;7:511-516.

- Meyerowitz EA, Richterman A, Gandhi RT, Sax PE: Transmission of SARS-CoV-2: A Review of Viral, Host, and Environmental Factors. Ann Intern Med 2021;174:69-79.

- O'Brien E, Xagoraraki I: A water-focused one-health approach for early detection and prevention of viral outbreaks. One Health 2019;7:100094.

- Oude Munnink BB, Sikkema RS, Nieuwenhuijse DF, Molenaar RJ, Munger E, Molenkamp R, et al. Transmission of SARS-CoV-2 on mink farms between humans and mink and back to humans. Science 2021;371:172-177.

- Paules CI, Marston HD, Fauci AS: Coronavirus Infections-More Than Just the Common Cold. JAMA 2020;323:707.

- Peccia J, Zulli A, Brackney DE, Grubaugh ND, Kaplan EH, Casanovas-Massana $A$, et al. Measurement of SARS-CoV-2 RNA in wastewater tracks community infection dynamics. Nat Biotechnol 2020;38:1164-1167.

- Peng L, Liu J, Xu W, Luo Q, Chen D, Lei Z, et al. SARS-CoV-2 can be detected in urine, blood, anal swabs, and oropharyngeal swabs specimens. J Med Virol 2020;92:1676-1680.

- Prado T, de Castro Bruni A, Barbosa MRF, Garcia SC, Moreno LZ, Sato MIZ: Noroviruses in raw sewage, secondary effluents and reclaimed water produced by sand-anthracite filters and membrane 
bioreactor/reverse osmosis system. Science of The Total Environment 2019;646:427-437.

- Prevost B, Lucas FS, Ambert-Balay K, Pothier P, Moulin L, Wurtzer S: Deciphering the Diversities of Astroviruses and Noroviruses in Wastewater Treatment Plant Effluents by a High-Throughput Sequencing Method. Appl Environ Microbiol 2015;81:7215-7222.

- Randazzo W, Truchado P, Cuevas-Ferrando E, Simón P, Allende A, Sánchez G: SARS-CoV-2 RNA in wastewater anticipated COVID-19 occurrence in a low prevalence area. Water Research 2020;181:115942.

- Rothe C, Schunk M, Sothmann P, Bretzel G, Froeschl G, Wallrauch C, et al. Transmission of 2019-nCoV Infection from an Asymptomatic Contact in Germany. N Engl J Med 2020;382:970-971.

- Schmitz BW, Innes GK, Prasek SM, Betancourt WQ, Stark ER, Foster AR, et al. Enumerating asymptomatic COVID-19 cases and estimating SARS-CoV-2 fecal shedding rates via wastewater-based epidemiology. Science of The Total Environment 2021;801:149794.

- Serra-Compte A, González S, Arnaldos M, Berlendis S, Courtois S, Loret JF, et al. Elimination of SARS-CoV-2 along wastewater and sludge treatment processes. Water Research 2021;202:117435.

- Sultan S, Altayar O, Siddique SM, Davitkov P, Feuerstein JD, Lim JK, et al. AGA Institute Rapid Review of the Gastrointestinal and Liver Manifestations of COVID-19, Meta-Analysis of International Data, and Recommendations for the Consultative Management of Patients with COVID-19. Gastroenterology 2020;159:320-334.e27.

- Tian Y, Rong L, Nian W, He Y: Review article: gastrointestinal features in
COVID-19 and the possibility of faecal transmission. Aliment Pharmacol Ther 2020;51:843-851.

- Varbanov M, Bertrand I, Philippot S, Retourney C, Gardette M, Hartard C, et al. Somatic coliphages are conservative indicators of SARS-CoV-2 inactivation during heat and alkaline $\mathrm{pH}$ treatments. Science of The Total Environment 2021;797:149112.

-V'kovski P, Kratzel A, Steiner S, Stalder $\mathrm{H}$, Thiel V: Coronavirus biology and replication: implications for SARS-CoV-2. Nat Rev Microbiol 2021;19:155-170.

- Wang W, Xu Y, Gao R, Lu R, Han K, Wu $\mathrm{G}$, et al. Detection of SARS-CoV-2 in Different Types of Clinical Specimens. JAMA 2020;323:1843-1844.

-Wild CP: Complementing the Genome with an "Exposome": The Outstanding Challenge of Environmental Exposure Measurement in Molecular Epidemiology. Cancer Epidemiology Biomarkers \& Prevention 2005;14:1847-1850.

- Wilder-Smith A, Chiew CJ, Lee VJ: Can we contain the COVID-19 outbreak with the same measures as for SARS? The Lancet Infectious Diseases 2020;20:e102-e107.

- Wölfel R, Corman VM, Guggemos W, Seilmaier M, Zange S, Müller MA, et al. Virological assessment of hospitalized patients with COVID-2019. Nature 2020;581:465-469.

- Wu Y, Guo C, Tang L, Hong Z, Zhou J, Dong $\mathrm{X}$, et al. Prolonged presence of SARS-CoV-2 viral RNA in faecal samples. The Lancet Gastroenterology \& Hepatology 2020;5:434-435.

- Wurtzer S, Maréchal V, Bertrand I, Boni M, Le Guyader S, Moulin L, et al. Viral infectious diseases seen through wastewater. Virologie 2021a;25:8-11.

- Wurtzer S, Marechal V, Mouchel J, Maday Y, Teyssou R, Richard E, et al.
Evaluation of lockdown effect on SARS-CoV-2 dynamics through viral genome quantification in waste water, Greater Paris, France, 5 March to 23 April 2020. Eurosurveillance 2020; 25. DOI: 10.2807/1560-7917.ES.2020.25.50.2000776 -Wurtzer S, Waldman P, Ferrier-Rembert A, Frenois-Veyrat G, Mouchel JM, Boni $\mathrm{M}$, et al. Several forms of SARS-CoV-2 RNA can be detected in wastewaters: Implication for wastewater-based epidemiology and risk assessment. Water Research 2021b;198:117183.

- Xiao F, Tang M, Zheng X, Liu Y, Li X, Shan $\mathrm{H}$ : Evidence for Gastrointestinal Infection of SARS-CoV-2. Gastroenterology 2020;158:1831-1833.e3.

- Xu Y, Li X, Zhu B, Liang H, Fang C, Gong Y, et al. Characteristics of pediatric SARS-CoV-2 infection and potential evidence for persistent fecal viral shedding. Nat Med 2020;26:502-505.

- Zhang Y, Cen M, Hu M, Du L, Hu W, Kim JJ, et al. Prevalence and Persistent Shedding of Fecal SARS-CoV-2 RNA in Patients With COVID-19 Infection: A Systematic Review and Meta-analysis. Clin Transl Gastroenterol 2021;12:e00343.

- Zhang Y, Chen C, Zhu S, Shu C, Wang D, Song J, et al. Isolation of 2019-nCoV from a Stool Specimen of a LaboratoryConfirmed Case of the Coronavirus Disease 2019 (COVID-19). CCDCW 2020;2:123-124.

- Zhu N, Zhang D, Wang W, Li X, Yang B, Song J, et al. A Novel Coronavirus from Patients with Pneumonia in China, 2019. N Engl J Med 2020;382:727-733.

- Zinsstag J, Crump L, Schelling E, Hattendorf J, Maidane YO, Ali $\mathrm{KO}$, et al. Climate change and One Health. FEMS Microbiology Letters 2018;365. DOI: 10.1093/femsle/fny085 